\title{
FERMILAB ELECTRON COOLING PROJECT: ENGINEERING ASPECTS OF COOLING SECTION
}

\author{
J. Leibfritz ${ }^{\dagger}$, K. Carlson, A.C. Crawford, A. Makarov, M. McGee, S. Nagaitsev, G. Saewert, \\ F. Saffrahn, A. Shemyakin, A. Warner, FNAL*, Batavia, IL 60510, USA
}

\section{Abstract}

The Fermilab Electron Cooling project involves interacting a $4.3 \mathrm{MeV}, 0.5 \mathrm{~A}$ DC electron beam with 8.9 $\mathrm{GeV} / \mathrm{c}$ antiprotons in the FNAL Recycler Ring. This interaction occurs through a 20-meter long cooling section consisting of 10 solenoid modules. This cooling process would lead to an increase in the Tevatron collider luminosity needed to support RunIIb parameters. There are several important engineering aspects of this cooling section including: solenoid design, vacuum system design, magnetic shielding, support system, and alignment methods. Details of the engineering issues related to each of these areas will be discussed.

\section{INTRODUCTION}

The goal of achieving the Tevatron luminosity of $5 \times 10^{32} \mathrm{~cm}^{-2} \mathrm{~s}^{-1}$ for RunIIb requires Electron Cooling in the Recycler Ring to provide an increased flux of antiprotons [1]. The plan is to provide cooling of the $8.9 \mathrm{GeV} / \mathrm{c}$ antiprotons with a $4.3 \mathrm{MeV}$ electron beam over a 20meter long section of the Recycler. Fig. 1 shows a schematic layout of the Electron Cooling system in the Recycler Ring.

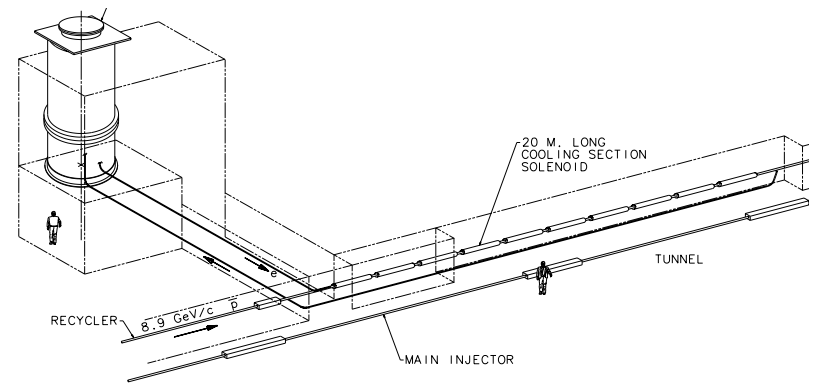

Figure 1: Schematic of FNAL Electron Cooling System

The cooling section is comprised of ten identical 2meter long solenoid modules. Each module consists of: a solenoid with end coil and dipole correctors, a vacuum system, magnetic shielding, and a support system. Fig. 2 shows a schematic of the cooling section module.

\section{SOLENOID DESIGN}

\subsection{General Design Parameters}

There are ten $74 "(188 \mathrm{~cm})$ long cooling section solenoids separated by a 6" $(15.4 \mathrm{~cm})$ gap for end coils and

\footnotetext{
*Operated by Universities Research Association, Inc., under Contract No. DE-AC02-76CH03000 with the U.S. Department of Energy

Leibfritz@fnal.gov
}

instrumentation. Table 1 summarizes the parameters of the solenoids. For a detailed description of the requirements for the cooling solenoids, see [2].

Table 1: Solenoid Module Parameters

\begin{tabular}{|l|l|}
\hline Solenoid Length & $74 "(188 \mathrm{~cm})$ \\
Weight & $550 \mathrm{lb} .(250 \mathrm{~kg})$ \\
Magnetic Field & $50-150 \mathrm{G}$ \\
Current for B=150 G & $4 \mathrm{~A}$ \\
Voltage & $80 \mathrm{~V}$ \\
\hline
\end{tabular}

\subsection{Solenoid Fabrication}

The construction of the solenoid begins with a spool consisting of a 6" diameter aluminum tube with disks welded to each end. The spool is wound with six layers of conductor. Insulated copper wire with a square crosssection (0.072" x 0.072") is used. A specially designed winding device maintains a tension on the conductor of 30 lbs. and also a spacing of no more than 0.005 " between adjacent turns. Each layer contains approximately 980 turns of conductor and is separated by two layers of 0.007 " thick dry fiberglass tape.

Once all six layers are wound, the spool assembly is inserted into the outer solenoid tube. The outer tube is an 8 " diameter aluminum tube with ribs welded around its circumference that serve a dual purpose. They provide channels for water-cooling as well as act as guides for positioning the magnetic shielding. The outer tube also has the support feet and alignment fixtures welded to it.

\subsection{Impregnation}

After the solenoid is assembled, it is impregnated in a vacuum oven with a heat cure epoxy. If done in a horizontal orientation, the inner tube is subjected to a considerable distributed load over its length due to the weight of the coil windings. Calculations show this load $(\approx 250 \mathrm{~kg}$ ) results in a deflection of $0.4 \mathrm{~mm}$. Because this deflection is present during the curing process, it becomes a permanent set in the magnet shape. Measurements of the first two prototype solenoids confirmed a permanent vertical deflection of $0.5 \mathrm{~mm}$.

As a result of this problem, a special curing fixture was developed so the solenoids could be impregnated in a vertical position, thus eliminating this deflection. This vertical curing fixture is currently being used to impregnate the first production solenoid. 


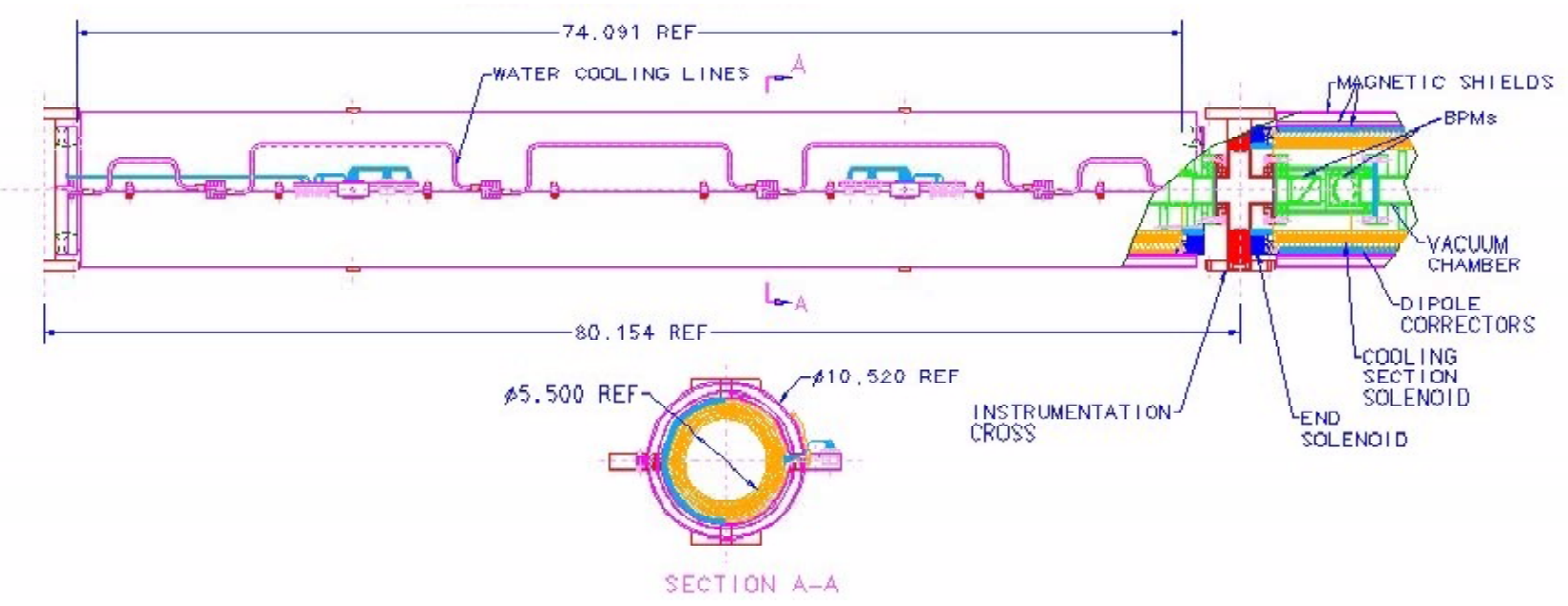

Figure 2: Schematic of Cooling Section Module

\subsection{End Coil Solenoids}

To help compensate for end effects through the instrumentation gaps between each solenoid, end coil solenoids are used at each end of the cooling solenoid assembly. As a result, the electron beam sees a more continuous homogenous field over the entire 20-meter cooling section [2].

The end coil solenoids have a diameter of approximately 8.5 " and are 1.9" wide. Their construction is similar to that of the cooling solenoids, except for the number of layers (22) and size of the conductor (0.05" diameter). Fig. 3 is a picture of the first production end coil solenoid.

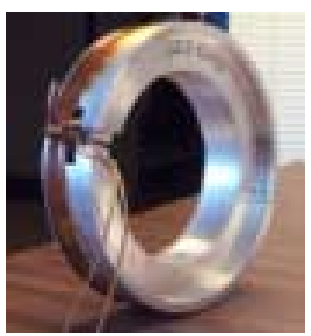

Figure 3: End Coil Solenoid

\subsection{Flexible Circuit Dipole Correctors}

Transverse field non-uniformities along the cooling section solenoid are caused by two major factors: mechanical sag and winding errors [3]. To compensate for these transverse deviations, eight dipole correction coil sets are used along each 2-meter solenoid. Each set is a pair of coils opposite the beam pipe in the $\mathrm{X}$ and $\mathrm{Y}$ transverse beam planes and is 9.125" in length. Calculations and measurements confirm that a field of 0.5 $\mathrm{G}$ in each coil is enough for correction, which enables the use of flexible printed circuit board technology to fabricate the coils.

The design of these coils optimized the width, thickness, and number of turns of the copper traces. The final design utilizes one amp of current and contains ten turns per coil. The copper traces are 0.015 " wide and have a spacing of $0.010 "$ ". Two-ounce copper on Kapton is the flexible circuit material. The flex circuits are wrapped around the outer solenoid tube and taped down with Kapton tape.

\subsection{Current Status of Solenoids}

Two prototype cooling solenoids were fabricated, installed, and measured in the Electron Cooling test facility at Fermilab, see Fig. 4. The results of these measurements show that the basic properties of the solenoids are satisfactory for the needs of the Electron Cooling project [4]. The work with these prototypes also uncovered some fabrication problems that have been addressed in the design of the final production solenoids.

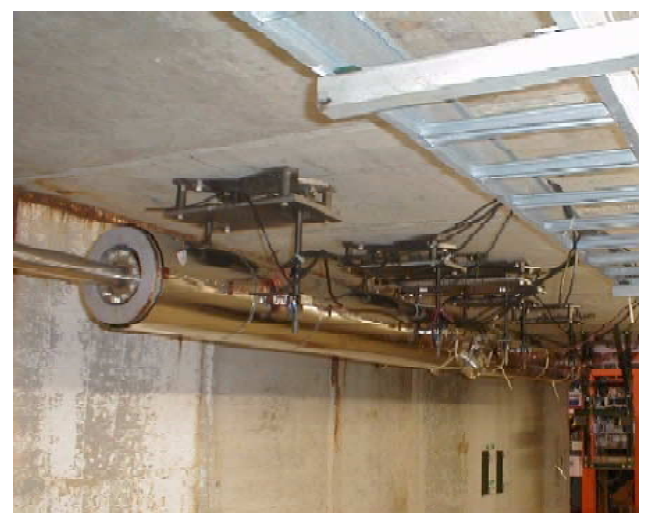

Figure 4: Prototype Electron Cooling Solenoids

The first of the final production solenoids is currently in the vacuum oven being impregnated and the second is being assembled. At a production rate of approximately two per month, all twelve (ten for the cooling section and two spares) solenoids should be done by November 2001.

\section{VACUUM SYSTEM DESIGN}

The vacuum system in the cooling section is made up of ten independent vacuum sections, which correspond to the ten cooling solenoids. This was done to simplify installation. Each vacuum section is approximately two 
meters in length and consists of a round beamtube, bellows, beam position monitor (BPM), and instrumentation cross (see Fig. 2).

The beamtube is a 2" diameter $316 \mathrm{~L}$ stainless steel tube. This material was selected to provide a system that is non-magnetic $(\mu \leq 1.05)$ and can achieve the ultra high vacuum required for insertion in Fermilab's Recycler Ring, specifically $1 \times 10^{-10}$ Torr. To help achieve this level of vacuum, the inside of the beamtube is electropolished and all components of the vacuum system are bakable to $150^{\circ} \mathrm{C}$.

The bellows provide for thermal movements of the tube, while a BPM and instrumentation cross are located in each vacuum section to provide diagnostic information at each solenoid. The cross is used to install two beam scrapers and attach an ion pump. The vacuum system design is nearly complete and fabrication is planned to begin soon.

\section{MAGNETIC SHIELDING}

The Electron Cooling section will be exposed to external transverse magnetic fields as high as 5 Gauss. These fields are due to several factors including current carrying busswork and residual magnetization of ferrous material in the beamline enclosure, as well as local components of the Earth field. It has been calculated that transverse momentum imparted to the electron beam by these magnetic fields is acceptably low if the fields can be limited to approximately 2 mGauss.

In order to accomplish this, the Electron Cooling section will be shielded by three concentric cylindrical layers of high initial permeability alloy. A geometry that achieves this goal and that fits within the radial limitations imposed by the solenoid design is parameterized in Table 2.

Table 2: Magnetic Shielding Parameters

\begin{tabular}{|l|l|}
\hline $\mathrm{Mu}$ (initial) & 11000 \\
Layer thickness & $1 \mathrm{~mm}$ \\
Inside radius of layer 1 & $109.5 \mathrm{~mm}$ \\
Inside radius of layer 2 & $120.6 \mathrm{~mm}$ \\
Inside radius of layer 3 & $133.3 \mathrm{~mm}$ \\
Total magnetic attenuation for DC fields & 3000 \\
\hline
\end{tabular}

Using an $80 \%$ Nickel-Iron-Molybdenum alloy satisfies this initial $\mathrm{Mu}$. It is necessary to anneal the shields in a hydrogen furnace after all forming processes to reach the high initial permeability. Tests have been conducted using this material and a design has been completed.

\section{SUPPORT AND ALIGNMENT}

\subsection{Support System}

The Electron Cooling support system will be mounted from the ceiling of the Recycler Ring enclosure. There are four support blocks welded at the $25 \%$ points of the outer solenoid tube, which have a 1.0" diameter hole for use in supporting and handling the solenoid

The initial design suspended the solenoid from above using four threaded rods attached to adjustable plates that provided the horizontal and lateral adjustment of \pm 1.0 " (see Fig. 4). Tests with the prototype solenoids found these supports were not rigid enough to eliminate small fluctuations in position. As a result, a 3-point gravity based stand is being developed which will use a spherical ball and socket arrangement; similar to the supports used throughout the Recycler Ring.

The instrumentation cross, located between adjacent solenoids, will have its own support system. This is done so the vacuum system can be aligned independently of the solenoids.

\subsection{Alignment}

The cooling section solenoids require an alignment precision of $0.2 \mathrm{~mm}$. The solenoid outer tube has four fiducials near each end, located $45^{\circ}$ radially apart. These fiducials contain a high precision hole used to hold the survey nest, which receives the laser tracker target used for alignment. The magnetic center of each solenoid will be measured and referenced to these fiducials with an accuracy of $0.1 \mathrm{~mm}$. The fiducials will then be used to align the solenoid to its desired position. The details of the alignment process of the solenoids and vacuum system are still being worked out.

\section{CONCLUSION}

The design of Fermilab's Electron Cooling section is proceeding well. The final production solenoids, end coils, and dipole correctors are being fabricated; the vacuum system and magnetic shielding designs are complete; and the support and alignment methods are being finalized. Tests of the entire cooling section will continue at the Electron Cooling test facility in preparation for installation in the Recycler Ring in 2003-2004.

\section{ACKNOWLEDGEMENTS}

The authors would like to thank L. Kozien and S. Wesseln for providing technical support and assistance with the development of this paper.

\section{REFERENCES}

[1] "Prospectus for an Electron Cooling System for the Recycler," Fermilab preprint FERMILAB-TM-2061, edited by J.A. MacLachlan, October 1998.

[2] S. Nagaitsev et al., "Cooling Section Solenoid for the $5 \mathrm{MeV}$ Fermilab Electron Cooling Project," EPAC'00, Vienna, June 2000.

[3] S. Nagaitsev, A. Shemyakin, and V. Vostrikov, Fermilab-FN-689, April 2000.

[4] A. Shemyakin et al., "Fermilab Electron Cooling Project: Field Measurements in the Cooling Section," these proceedings. 